\title{
AVALIAÇÃO DO EFEITO DO USO DE RACTOPAMINA SOBRE A QUALIDADE DA CARCAÇA E O RENDIMENTO DE CORTES SUÍNOS
}

\author{
D. S. MARTINS ${ }^{1}$, M. U. JANECZKO ${ }^{1}$, J. KILIAN ${ }^{1}$, J. STEFFENS ${ }^{1}$ e M. B. A. SOARES ${ }^{1}$ \\ ${ }^{1}$ Universidade Regional Integrada do Alto Uruguai e das Missões, Departamento de \\ Ciências Agrárias \\ E-mail para contato: daniela_s_martins@yahoo.com.br
}

\begin{abstract}
RESUMO - A carne suína é consumida mundialmente, e estudos contínuos são desenvolvidos para atender as necessidades de consumidores. O uso de Ractopamina na dieta animal promove o incremento da quantidade de carne magra em detrimento da quantidade de gordura na carne tornou-se uma prática cada vez mais adotada pelos produtores. O trabalho visa avaliar a influência do uso de Ractopamina sobre o rendimento e qualidade da carne suína. Utilizou-se 7,4 ppm de Ractopamina na dieta animal frente à não utilização do aditivo. Mostrou-se uma redução no rendimento de toucinho de paleta $(7,32 \%)$, toucinho de carré $(11,85 \%)$ e barriga $(2,34 \%)$. Porém, a perda de peso da carcaça e o rendimento dos cortes inteiros de pernil, paleta e sobrepaleta não foram influenciados. Nos cortes do padrão comercial (sem ossos e gorduras) observou-se maior rendimento de pernil $(7,45 \%)$ e paleta $(8,77 \%)$. Na qualidade da carne, não influenciou sobre a cor e capacidade de retenção de água, mas teve influência sobre a redução do marmoreio da carne. $\mathrm{O}$ pH final da carne $(6,0)$ foi caracterizado como normal. Conclui-se que a utilização de Ractopamina não exerce influência negativa sobre a qualidade da carne suína.
\end{abstract}

Palavras-chave: Ractopamina, rendimento, qualidade e carne suína.

\section{INTRODUÇAO}

A carne suína é a fonte de proteína animal mais consumida no mundo, sendo aproximadamente $77 \%$ superior ao volume de carne bovina e $25 \%$ superior ao volume de carne de frango (ABIPECS, 2012; FAO, 2011).

Estudos e investimentos em suinocultura (sanidade, nutrição, bom manejo, produção integrada, capacitação de produtores) contribuíram para aumentar a oferta interna e colocar o Brasil em destaque no cenário mundial, estando posicionado em quarto lugar no ranking de produção e exportação mundial de carne suína (ABIPECS, 2012; MAPA, 2012).

O mercado consumidor de alimentos, a nível mundial, está cada vez mais preocupado com os aspectos relacionados à saúde, sendo que a carne gorda aparece em muitos casos como sinônimo de baixa qualidade. Especialistas brasileiros investiram na evolução genética de suínos por 20 anos, o que reduziu em 31\% a gordura da carne, 10\% do colesterol e 14\% de calorias (MAPA, 2012). Outra maneira de reduzir o conteúdo de gordura e promover o 
incremento de carne na carcaça é a utilização de aditivos alimentares na dieta nutricional dos animais, com destaque para os repartidores de energia, a exemplo da Ractopamina.

A ractopamina foi aprovada para uso no Brasil em 1996 e desde então seu efeito vem sendo amplamente estudado e seu uso disseminado para melhorar a qualidade da carne suína, especificamente a redução do conteúdo lipídico da carcaça (SANCHES, 2009), e a conversão alimentar (SANCHES, 2009).

A maior parte dos países importadores da carne brasileira permite o uso de ractopamina na dieta animal, porém a Rússia, segundo maior importador de carne brasileira, os países do Bloco Europeu e também a China, maior produtor de carne suína mundial, são extremamente exigentes quanto à garantia de ausência do uso de aditivos repartidores de energia na dieta animal.

Neste sentido, este trabalho tem por objetivo geral estudar a influência do uso de Ractopamina na dieta animal sobre a qualidade da carne da carcaça suína (classificação em carne PSE, DFD ou normal) e sobre o rendimento dos cortes, através da verificação dos parâmetros de qualidade da carcaça: $\mathrm{pH}$, cor, marmoreio (gordura intramuscular) e capacidade de retenção de água (expressa em perda de água por gotejamento - drip loss); da avaliação da perda de peso no resfriamento (rendimento de carcaça fria sobre carcaça quente); do rendimento dos cortes brutos: pernil, paleta (subdividida em toucinho da paleta e paleta sem toucinho), sobrepaleta, carré (subdividido em toucinho do carré e carré sem toucinho), e barriga com costela, sobre carcaça quente; e da avaliação do rendimento dos cortes de carne suína padrão exportação (pernil e paleta) e da gordura resultante da preparação destes cortes, todas medidas para carcaças de suínos que utilizaram e não utilizaram ractopamina na dieta.

\section{MATERIAIS E MÉTODOS}

Os experimentos foram realizados utilizando suínos machos castrados, tratados com diferentes tipos de ração na fase de terminação, sendo chamado de Tratamento 1 para os suínos que receberam Ractopamina na dieta (7,5 ppm por 25 dias) e Tratamento 2 para os suínos que não receberam Ractopamina. Os animais abatidos respeitando as normas de Abate Humanitário e as carcaças, após tipificadas, foram resfriadas em câmara de equalização com temperatura de aproximadamente $2 \pm 2^{\circ} \mathrm{C}$ por 24 horas e posteriormente conduzidas para a etapa de desossa.

Para os testes de rendimento das partes principais inteiras (com osso e com pele) da carcaça, foram utilizadas 50 carcaças ( 25 de cada tratamento). Os cortes foram obtidos através do fracionamento da carcaça nas partes brutas, sendo elas: pernil, paleta, sobrepaleta, barriga com costela e carré. Os resultados foram expressos pela relação de peso da parte inteira obtida no momento que antecede a desossa, 24 horas após o abate, pelo peso da carcaça quente (PQ) obtido no momento da tipificação (KUTZLER et al., 2011).

Nos testes de rendimento de cortes padrão exportação foram utilizadas 30 carcaças, sendo 15 de cada tratamento. A preparação dos cortes foi realizada pela separação das porções de pele, gordura, músculo e ossos, até a obtenção do padrão do corte comercial de Pernil e 
Paleta. Os resultados foram expressos pela relação de peso do corte preparado no padrão comercial com relação ao peso da parte inteira obtida no momento que antecede a desossa (desmembramento das partes: Paleta sem Toucinho, $\mathrm{P}_{\mathrm{PALS} / \mathrm{TOUC}}$ e Pernil sem pele, $\mathrm{P}_{\text {ER S/PELE}}$ ).

A qualidade da carcaça pode ser avaliada através de análise dos parâmetros de $\mathrm{pH}$, cor, marmoreio e capacidade de retenção de água, medidas em pontos específicos da carcaça, que são utilizados para estimar a qualidade da carcaça como um todo. Com exceção da análise do $\mathrm{pH}$, que pode ser medida tanto no músculo Semimembranosus (pernil) quanto no Longissimus dorsi (lombo, na altura da última costela), todas as outras medidas para avaliar a qualidade da carcaça são realizadas em amostra do músculo Longissimus dorsi (BRIDI \& SILVA, 2009).

A medida do pH foi realizada 1 hora após abate e 24h após o abate (BRIDI et al., 2003, HINSON et al., 2011) a partir de amostra de pernil (Semimembranosus), utilizando equipamento de pH com eletrodo de inserção ( $\mathrm{pH}$ 320/Ste-1, WTW). Foram realizadas 5 coletas e a análise realizada em triplicata para cada tratamento.

Após 24 horas de resfriamento da carcaça na câmara frigorífica entre 0 e $2^{\circ} \mathrm{C}$, foram coletadas cinco amostras de aproximadamente $1 \mathrm{~cm}$ de espessura do músculo lombo (Longissimus dorsi) a partir da articulação da última vértebra torácica com a primeira vértebra lombar (adaptado de BRIDI et al., 2003), para a realização das avaliações de cor, marmoreio, e capacidade de retenção de água (CRA, drip loss).

Antes da avaliação da cor e marmoreio, as amostras permaneceram expostas ao ar (20 minutos) para permitir a oxigenação do músculo e com o auxílio de padrões fotográficos (National Pork Producers Council, 1989) atribuíram-se valores de para cor de1 = rosa muito pálido a $6=$ vermelho muito escuro (BRIDI et al., 2003; TONIETI, 2008). A avaliação do marmoreio foi realizado com o auxílio de padrões fotográficos (National Pork Producers Council, 1989) em que foram atribuidos valores para o marmoreio (gordura intramuscular) de $1=$ sem gordura intramuscular a $10=$ extrema gordura intramuscular (BRIDI et al., 2003; TONIETI, 2008).

Para a avaliação da capacidade de retenção de água, medida através da perda de água por gotejamento, foram separadas amostras de aproximadamente $35 \mathrm{~g}$ de lombo que foram identificadas, pesadas em balança semi-analítica e suspensas por ganchos metálicos e, individualmente, colocadas dentro de saco de polietileno (dupla camada) e fechados com barbante. As amostras permaneceram penduradas por 60 horas (2,5 dias) refrigeradas a $3 \pm$ $2^{\circ} \mathrm{C}$. Ao final deste tempo, elas foram retiradas da embalagem plástica e, antes da pesagem final, foi removida a umidade superficial com auxílio de papel absorvente (adaptação de BRIDI et al., 2003; MOORE et al., 2012; WARRIS et al., 1990 e TONIETI, 2008). A perda de água por gotejamento (Drip loss) foi expressa pela diferença entre o peso da amostra no final do processo pelo peso inicial.

Os resultados foram analisados estatisticamente por Análise de Variância (ANOVA) e as médias comparadas pelo teste de Tukey, com 95\% de confiança. 


\section{RESULTADOS E DISCUSSÃO}

O rendimento das partes inteiras componentes da carcaça foi obtido através do fracionamento da carcaça nas partes bruta e descrito como $R_{P E R}$ (Rendimento de Pernil), $R_{P A L}$ (Rendimento de Paleta, subdividido em rendimento da paleta sem toucinho, RPAL S/TOUC, e toucinho da paleta, RTouc. PaL), $R_{\text {SP }}$ (Rendimento de Sobrepaleta), $R_{B A R}$ (Rendimento de Barriga com Costela), $\mathrm{R}_{\mathrm{CAR}}$ (Rendimento de Carré, subdividido em rendimento de Carré sem toucinho, RCAR S/TOUC, e toucinho do carré, RTOUC. CAR). Os resultados de média de rendimento dos cortes nos diferentes tratamentos, desvio padrão e nível de significância (p-valor) estão mostrados na Tabela 1.

Tabela 1 - Efeito do uso de Ractopamina sobre o rendimento dos Cortes inteiros sobre carcaça quente: média, desvio padrão e nível de significância.

\begin{tabular}{ccccc}
\hline \multirow{2}{*}{ Tipos de Cortes } & \multicolumn{2}{c}{$1-$ Com RAC $(\%)$} & \multicolumn{2}{c}{$2-$ Sem RAC $(\%)$} \\
\cline { 2 - 5 } & Média & Desvio Pad. & Média & Desvio Pad. \\
\hline R $_{\text {PER }}$ & $25,78^{\mathrm{a}}$ & $\pm 0,88$ & $25,81^{\mathrm{a}}$ & $\pm 1,12$ \\
$\mathrm{R}_{\text {PAL }}$ & $17,78^{\mathrm{b}}$ & $\pm 0,82$ & $17,96^{\mathrm{b}}$ & $\pm 0,67$ \\
$\mathrm{R}_{\text {PAL S/TOUC }}$ & $14,95^{\mathrm{c}}$ & $\pm 0,92$ & $14,91^{\mathrm{c}}$ & $\pm 0,77$ \\
$\mathrm{R}_{\text {TOUC. PAL }}$ & $2,83^{\mathrm{d}}$ & $\pm 0,47$ & $3,05^{\mathrm{e}}$ & $\pm 0,44$ \\
$\mathrm{R}_{\text {SOBREP }}$ & $8,61^{\mathrm{f}}$ & $\pm 0,77$ & $8,82^{\mathrm{f}}$ & $\pm 0,52$ \\
$\mathrm{R}_{\text {BAR }}$ & $18,93^{\mathrm{g}}$ & $\pm 0,80$ & $18,50^{\mathrm{h}}$ & $\pm 0,74$ \\
$\mathrm{R}_{\text {CAR }}$ & $22,47^{\mathrm{i}}$ & $\pm 0,83$ & $22,15^{\mathrm{i}}$ & $\pm 1,02$ \\
$\mathrm{R}_{\text {CAR S/ TOUC. }}$ & $19,45^{\mathrm{j}}$ & $\pm 0,78$ & $18,72^{\mathrm{k}}$ & $\pm 0,95$ \\
$\mathrm{R}_{\text {TOUC. CAR }}$ & $3,02^{1}$ & $\pm 0,29$ & $3,43^{\mathrm{m}}$ & $\pm 0,27$ \\
\hline
\end{tabular}

* Letras iguais na mesma linha, não diferem estatisticamente ao nível de 5\%.

Conforme observado na Tabela 1, não há interferência significativa do uso de Ractopamina (7,5 ppm) sobre o rendimento dos cortes inteiros de pernil, paleta inteira, paleta sem toucinho, sobrepaleta e carré inteiro, porém há redução no rendimento de toucinho da paleta e toucinho do carré no tratamento com ractopamina em comparação ao tratamento sem o aditivo e aumento no rendimento de barriga com costela e de carré sem toucinho no tratamento com ractopamina em comparação ao tratamento sem ractopamina. Kutzler et al. (2011) não observou diferença significativa entre rendimento de cortes de suínos tratados com $6,2 \mathrm{ppm}$ de ractopamina frente aos tratamentos controles sem ractopamina, entretanto, Carr, et al. (2009) observou uma diferença significativa no incremento no rendimento de pernil, sobrepaleta, barriga e lombo nos tratamentos com 5 ppm.

Os resultados de rendimento dos cortes padrão exportação foram expressos como Rendimento de Pernil: $R_{\text {PERnIL }}$, Rendimento de Paleta: $R_{\text {PALETA }}$ e Rendimento da Gordura do Pernil e da Paleta: $R_{\text {GORDURA, e podem ser observados na Tabela } 2 .}$

O rendimento da paleta padrão comercial foi em média 9,03\% maior nas carcaças de suínos tratados com ractopamina, ao mesmo tempo em que o rendimento das gorduras foi em média 20,59\% menor neste mesmo tratamento, o que indica que a utilização do aditivo interfere sobre a quantidade de carne no corte de paleta. Carr et al. (2009) somente observou aumento de carne na paleta a partir do uso de 20 ppm de ractopamina. 
Tabela 2 - Efeito do uso de Ractopamina sobre o rendimento de cortes padrão exportação e gordura.

\begin{tabular}{ccccc}
\hline \multirow{2}{*}{ Tipos de corte } & \multicolumn{2}{c}{$1-$ Com RAC } & \multicolumn{2}{c}{ 2 - Sem RAC } \\
\cline { 2 - 5 } & Média & Desvio Pad. & Média & Desvio Pad. \\
\hline Paleta & & & & \\
R $_{\text {PALETA CORTE COMERCIAL }}$ & $57,02 \%^{\mathrm{a}}$ & $\pm 3,92 \%$ & $52,29 \%$ & $\pm 3,87 \%$ \\
R $_{\text {GORDURA DA PALETA }}$ & $8,56 \%^{\mathrm{c}}$ & $\pm 1,90 \%$ & $10,77 \%^{\mathrm{d}}$ & $\pm 3,54 \%$ \\
Pernil & & & & \\
$\mathrm{R}_{\text {PERNIL CORTE COMERCIAL }}$ & $64,67 \%^{\mathrm{e}}$ & $\pm 1,10 \%$ & $60,18 \%^{\mathrm{f}}$ & $\pm 1,40 \%$ \\
$\mathrm{R}_{\text {GORDURA DO PERNIL }}$ & $11,46 \%^{\mathrm{g}}$ & $\pm 1,53 \%$ & $12,24 \% \mathrm{~g}$ & $\pm 1,96 \%$ \\
\hline
\end{tabular}

Rendimento \% com base no peso de paleta em toucinho e peso de pernil sem pele; dados referentes às outras partes (pele, ossos, músculo) não estão mostrados.

"Letras iguais na mesma linha, não diferem estatisticamente ao nível de 5\%.

O rendimento do pernil padrão comercial foi 7,46\% maior nas carcaças de suínos tratados com ractopamina. Resultados similares foram obtidos por Kutzler et al. (2011).

$\mathrm{O}$ uso de ractopamina não apresentou efeito significativo sobre o $\mathrm{pH}$ inicial, drip loss e cor, porém o tratamento com o aditivo apresentou maior $\mathrm{pH}$ final e menor quantidade de gordura marmorizada do que tratamento sem o uso RAC. Os resultados das análises de qualidade da carne (pH, cor, marmoreio e capacidade de retenção de água) estão apresentados na Tabela 3.

Tabela 3 - Efeito do uso de Ractopamina sobre as características de qualidade da carne: média, desvio padrão e nível de significância.

\begin{tabular}{ccccc}
\hline \multirow{2}{*}{ Medida } & \multicolumn{2}{c}{1 - Com RAC } & \multicolumn{2}{c}{2 - Sem RAC } \\
\cline { 2 - 5 } & Média & Desvio Pad. & Média & Desvio Pad. \\
\hline pH inicial $^{1}$ & $6,38^{\mathrm{a}}$ & $\pm 0,23$ & $6,33^{\mathrm{a}}$ & $\pm 0,20$ \\
$\mathrm{pH} \mathrm{final}^{2}$ & $5,88^{\mathrm{b}}$ & $\pm 0,07$ & $5,61^{\mathrm{c}}$ & $\pm 0,08$ \\
Drip loss $(\%)$ & $4,59 \%^{\mathrm{d}}$ & $\pm 0,96 \%$ & $3,82 \%^{\mathrm{d}}$ & $\pm 1,83 \%$ \\
Cor & $3,17^{\mathrm{e}}$ & $\pm 0,41$ & $3,33^{\mathrm{e}}$ & $\pm 0,52$ \\
Marmoreio $^{\mathrm{f}}$ & $2,00^{\mathrm{f}}$ & $\pm 0,00$ & $2,67^{\mathrm{g}}$ & $\pm 0,52$ \\
\hline
\end{tabular}

${ }^{1} \mathrm{pH}$ inicial medido $1 \mathrm{~h}$ após abate

${ }^{2} \mathrm{pH}$ final 24h após abate

"Letras iguais na mesma linha, não diferem estatisticamente ao nível de 5\%.

$\mathrm{O} \mathrm{pH}$ final ficou mais elevado da carcaça tratada com Ractopamina, efeito semelhante no $\mathrm{pH}$ final foi observado por Warriss et al. (1990), indicando que o uso de beta adrenérgico tendia a esgotar o glicogenio no fígado e músculos e com isto o $\mathrm{pH}$ final se eleva e reduz a saturação de cor do músculo. Da mesma forma Hinson et al. (2011) observou diferença significativa no $\mathrm{pH} 24 \mathrm{~h}$, entre carcaças tratadas com 7,4 ppm de ractopamina ( $\mathrm{pH} 5,88)$ frente aos animais tratados sem o aditivo $(\mathrm{pH} 5,74)(\mathrm{p}<0,05)$. Em contrapartida, Kutzler et al. (2011) e Boler et al. (2011) não observaram diferença significativa no $\mathrm{pH} 24 \mathrm{~h}$ entre suínos tratados com 6,2 e 7,4 ppm de ractopamina, respectivamente, frente aos tratamentos controles sem o aditivo. 
A cor obtida para ambos os tratamentos realizados neste estudo encontra-se dentro da faixa considerada normal na carne suína, que segundo Bridi et al. (2003) é igual a três, que corresponde à cor rosa acinzentado (NPPC, 1989). Hinson et al. (2011) observou impacto significativo do uso de ractopamina $(\mathrm{p}<0,05)$ sobre a cor, em que o tratamento com $7,4 \mathrm{ppm}$ de ractopamina apresentou escores menores para a cor. Em contrapartida, Moore et al. (2012), Gonzalez et al. (2010) e Fernandez-Duenas et al. (2008) não observaram diferença na cor da carne em animais tratados com ractopamina frente aos tratamentos controles.

Similar ao observado na avaliação do marmoreio do presente trabalho, Gonzalez et al. (2010), Agostini (2008) e Armstrong et al. (2004) observaram redução significativa no marmoreio das carcaças tratadas com ractopamina frente ao controle sem o aditivo. Diferentemente ao observado no presente estudo, Kutzler et al. (2011), Hinson et al. (2011) e Moore et al. (2012) não observaram diferença nas avaliações de marmoreio frente aos tratamentos sem o aditivo.

Com relação à perda de água por gotejamento, assim como o observado no presente estudo, Moore et al. (2012) e Hinson et al. (2011) não observaram diferença significativa na perda de água por gotejamento (drip loss) no tratamento com ractopamina frente ao controle, Moore et al. (2012) obteve médias de 3,26\% e 3,58\% e Hinson et al. (2011) obteve médias de $6,21 \%$ e $5,11 \%$ para os tratamentos controle e com ractopamina, respectivamente.

A análise dos parâmetros de qualidade da carne indicou não haver diferença na classificação da carne dos dois tratamentos, ficando ambos nos parâmetros de carne Normal, conforme classificação de Bridi e Silva (2009). Da mesma forma, Braña et al. (2013) não observaram efeito da administração de $5 \mathrm{ppm}$ de ractopamina sobre a qualidade da carne, frente ao tratamento sem o aditivo.

\title{
4. CONCLUSÃO
}

Desta forma, se pode concluir que a utilização de ractopamina na dieta tem ação sobre o rendimento dos cortes padrão comercial e redução da gordura na carcaça, não exercendo influência negativa sobre a qualidade da carne suína.

\section{REFERÊNCIAS BIBLIOGRÁFICAS}

\author{
ABIPECS, ASSOCIAÇÃO BRASILEIRA DA INDÚSTRIA PRODUTORA E \\ EXPORTADORA DE CARNE SUÍNA. 2012; 2013. Disponível \\ em:<http://www.abipecs.org.br> Acesso em: 20/5/2012 e 08/9/2013.
}

AGOSTINI, P. S. Ractopamina para suínos: efeitos no desempenho, qualidade de carcaça e carne, parâmetros sanguíneos e comportamento. Dissertação (Mestrado em Ciência de Alimentos) - Programa de Mestrado e Doutourado em Ciência de Alimentos, Universidade Federal de Londrina, Londrina, 2008.

ARMSTRONG, T. A.; IVERS, D. J.; WAGNER, J. R.; ANDERSON, D. B.; WELDON, W. C.; BERG, E. P. The effect of dietary ractopamine concentration and duration of feeding on 
growth performance, carcass characteristics, and meat quality of finishing pigs. Journal of Animal Science, n.82, p. 3245-3253. 2004.

BRAÑA, D. V.; ROJO GOMEZ, G. A.; ELLIS, M.; CUARON, J. A. Effect of gender (gilt, surgically- and immuno-castrated male) and ractopamine hydrochloride supplementation on growth performance, carcass and pork quality characteristics of finishing pigs under commercial conditions. Journal of Animal Science, n. 91, p.6546-6556. 2013.

BRIDI A. M. RÜBENSAM, J. M.; NICOLAIEWSKY, S.; FELIX LOPES, R. F. F.; LOBATO, J. F. P. Efeito do Genótipo Halotano e de Diferentes Sistemas de Produção na Qualidade da Carne Suína. Revista Brasileira de Zootecnia, v.32, n.6, p.1362-1370, 2003.

BRIDI, A. M.; SILVA, C. A. Avaliação da Carne Suína. Midiograf, $1^{\circ}$ ed. 120p. Londrina, 2009.

CARR, S. N.; HAMILTON, D. N.; MILLER, K. D.; SCHROEDER, A. L.; FERNÁNDEZDUEÑAS, D.; KILLEFER, J.; ELLIS, M.; MCKEITH, F. K. The effect of ractopamine hydrochloride (Paylean) on lean carcass yields and pork quality characteristics of heavy pigs fed normal and amino acid fortified diets. Meat Science, n.81, p. 533-539, 2009.

FAO, Food and Agriculture Organization of the United Nations. Mapping supply and demand for animal-source foods to 2030. Roma. 2011. Disponível em <http://www.fao.org/docrep/014/i2425e/i2425e00.htm> Acesso em 08/9/2013.

FERNANDEZ-DUENAS, D. M.; MYERS, A. J.; SCRAMLIN, S. M.; PARKS, C. W.; CARR, S. N.; KILLEFER, J.; MCKEITH, F. K. Carcass, meat quality, and sensory characteristics of heavy body weight pigs fed ractopamine hydrochloride (Paylean). Journal of Animal Science. n. 86, p.3544-3550, 2008.

GONZALEZ, J. M.; JOHNSON, S. E.; STELZLENI, A. M.; THRIFT, T. A.; SAVELL, J. D.; WARNOCK, T. M.; JOHNSON, D. D. Effect of ractopamine- $\mathrm{HCl}$ supplementation for 28 days on carcass characteristics, muscle fiber morphometrics, and whole muscle yields of six distinct muscles of the loin and round. Meat Science, n.85, p.379-384, United States, 2010.

HINSON, R. B.; WIEGAND, B. R.; RITTER, M. J.; ALLEE, G. L.; CARR, S. N. Impact of dietary energy level and ractopamine on growth performance, carcass characteristics, and meat quality of finishing pigs. Journal of animal science. n. 89, p.3572-3579, 2011.

KUTZLER, L. W.; HOLMER, S. F.; BOLER, D. D.; CARR, S. N.; RITTER, M. J.; PARKS, C. W.; MCKEITH, F. K.; KILLEFER, J. Comparison of varying doses and durations of ractopamina hydrochloride on late-finishing pig carcass characteristics and meat quality. Journal of Animal Sciences, n.89, p.2176-2188, 2011.

MAPA: MINISTÉRIO DA AGRICULTURA, PECUÁRIA E ABASTECIMENTO. Disponível em: $<$ http://www.agricultura.gov.br/animal/especies/suinos $>$. Acesso em: 09 abr. 2012.

MOORE, K. L.; MULLAN, B. P.; D'SOUZA, D. N. The interaction between ractopamine supplementation, porcine somatotropin and moisture infusion on pork quality. Meat Science, Austrália, n. 92, p. 125-131, 2012.

NPPC. Pork Quality Standards. National Pork Producers Council in cooperation with National Pork Board 4/99.04037, 1989. 
SANCHES, J. F. Níveis de Ractopamina nas dietas de suínos machos castrados na fase de terminação. 2009. 56 f. Dissertação (Mestrado em Ciência Animal) - Programa de Mestrado em Ciência Animal, Faculdade de Medicina Veterinária e Zootecnia, Universidade Federal do Mato Grosso do Sul, Campo Grande, 2009.

TONIETI, A. P. Avaliações do desempenho zootécnico, qualidade da carcaça e carne em suíno macho inteiro imunocastrado. 2008. 129 f. Dissertação (Mestrado em Ciência) Programa de Mestrado em Ciência, Escola Superior de Agricultura Luiz de Queiroz, São Paulo, Piracicaba, 2008.

WARRISS, P. D.; BROWN, S. N.; ROLPH, T. P.; KESTIN, S. C. Interactions between the beta-adrenergic agonist salbutamol and genotype on meat quality in pigs. Journal of Animal Science, Philadelphia, E.U.A., v. 68, n. 11, p. 3669-3676, 1990. 